\title{
Convective Flow of Blood in Square and Circular Cavities
}

\author{
P. Senel and M. Tezer-Sezgin
}

\begin{abstract}
In this study, the fully developed, steady, laminar flow of blood is studied in a long pipe with square and circular cross-sections subjected to a magnetic field generated by an electric wire. Temperature difference between the walls causes heat transfer within the fluid by the displacement of the magnetizable fluid particles in the cavity. The governing equations are the coupled Navier-Stokes and energy equations including magnetization terms. The axial velocity is also computed with the obtained plane velocity. The Dual Reciprocity Boundary Element Method (DRBEM) is used by taking all the terms other than Laplacian as inhomogeneity which transforms the partial differential equations into the boundary integral equations. Numerical results are given for increasing values of Magnetic $(M n)$ and Rayleigh $(R a)$ numbers. The numerical results reveal that an increase in $M n$ accelerates the plane velocity in the cavity but decelerates the axial velocity around the magnetic source. Pressure increases through the channel starting from the magnetic source. Isotherms show the cooling of the channel with high $M n$ and $R a$ only leaving a thin hot layer near the top heated wall. As $R a$ increases viscous effect is reduced leaving its place to convection in the channel. The use of DRBEM has considerably small computational expense compared to domain type methods.
\end{abstract}

Key Words: Biomagnetic fluid, heat transfer, magnetic field, DRBEM Received: November, 2016.

Revised: March, 2017.

Accepted: June, 2017. 


\section{Introduction}

Biomagnetic fluid dynamics (BFD) is the area, investigating the biological fluid in the presence of magnetic fields. The most characteristic biomagnetic fluid is blood. Blood is a magnetizable fluid due to the cell membrane and the hemoglobin molecule, iron-based protein, carried by the red blood cells [1]. An externally applied magnetic field changes the flow and the heat transfer characteristics of blood significantly, resulting in some applications in biomedicine as cell separation, drug targeting, magnet therapies and controlling bleeding during surgeries. Tzirtzilakis et al. [2] investigated the fully developed blood flow in a square cavity. They solved the governing equations with a pressurelinked pseudotransient method. Biomagnetic fluid flow model is extended by taking into account both magnetization and Lorentz forces by Tzirtzilakis [3]. Kenjeres [4] carried the blood flow analysis to realistic arteries. He reported that an imposed non-uniform magnetic field could have created significant changes in the secondary flow patterns which made it possible to optimize the targeted drug delivery. Khashan et al. [5] presented a numerical simulation for magnetically mediated separation of labeled biospecies from a native fluid flowing through a two-dimensional channel using finite volume method. The influence of a dipole like field and a magnetic field generated by a thin electric wire on the flow of biomagnetic fluid flow in a circular duct with stenosis is studied by Tzirakis et al. [6]. They have used a method based on pressure correction scheme combining discontinuous and continuous Galerkin approximations.

The heat transfer through the blood flow in the presence of magnetic field can be used in hypothermic sessions, thermal simulation and thermal therapy applications [7]. The stretching disk flow of a heated biomagnetic fluid is investigated as a special case by Tzirtzilakis and Kafoussias [8]. They transformed the nonlinear system of equations to ordinary differential equations by introducing appropriate non-dimensional variables. The blood flow between two parallel plates is numerically simulated by Loukopoulos and Tzirtzilakis [9] using a finite difference scheme. They reported that the temperature and the rate of heat transfer are increasing the area where the magnetic source is placed. A finite element study of flow and temperature disturbance between two parallel plates subjected to multiple point magnetic sources is presented by Morega and Four [10]. Alimohamadi et al. [11] investigated the influence of numerous numbers of magnetic dipoles on the heat transfer in a rectangular duct. They compared MHD (Magnetohydrodynamics) and FHD (Ferrohydrodynamics) analysis of the flow which are obtained by a commercial software. The gravitational acceleration effect on the flow and heat transfer of blood flow between parallel plates is studied by Idris et al. [12] using a finite difference 
method.

The Dual Reciprocity Boundary Element Method (DRBEM) is essentially a generalized way of constructing particular solutions to the inhomogeneity of the differential equation that can be used to solve non-linear and time dependent problems [13]. Senel and Tezer-Sezgin [14] presented DRBEM solutions of Stokes and Navier-Stokes equations subjected to spatially varying point source magnetic field in circular and rectangular lid-driven cavities. Biomagnetic fluid flow between parallel plates imposed to a magnetic source was investigated by Tezer-Sezgin et al. [15]. They used both finite element and dual reciprocity boundary element method for solving momentum and energy equations in terms of stream function and vorticity. Recently, Senel and Tezer-Sezgin [16] studied the forced convection biomagnetic fluid flow in a square cavity.

In this paper, the fully developed, steady, flow of blood in the cross-section of a long pipe (cavity) under the influence of magnetization and buoyancy forces are investigated. The flow configuration, pressure and the temperature disturbance are visualized for various values of magnetic and Rayleigh numbers in square and circular cavities. The axial velocity profile is also presented. Viscous dissipation effect on the flow and the heat transfer is studied. To the best of authors' knowledge this is the first application of DRBEM to the mathematical model for the blood flow in square and circular cavities in primitive variables. DRBEM has the advantage of discretizing only the boundary of the cavity and results in considerably low computational expense.

\section{Mathematical Formulation}

Consider a fully developed, steady flow of biofluid in a long impermeable pipe with square or circular cross-section. The fluid is flowing through the axis of the pipe due to an imposed constant pressure gradient $P_{z}$ in the same direction. The flow and the heat transfer in the pipe are also affected by magnetization and buoyancy forces. Magnetization force is generated by a long wire carrying electric current placed $\bar{c}$ units below and parallel to the axis of the pipe. Buoyancy force occurs due to the temperature differences between the walls of the pipe and the gravitational acceleration. Being a hydrodynamically and thermally fully developed flow the problem is considered in the two-dimensional cross-section of the pipe (cavity) and in this case the electric wire serves as a point magnetic source. There is a longitudinal heat transport, but isotherms have the same profile on each cross-section of the pipe [17]. Magnetization, buoyancy forces and the constant pressure gradient in the axial direction develops a forced convection flow in the cavity. The axial velocity can be separately obtained with the computed plane velocity components. The governing equations in dimensional form are given by continuity, Navier- 
Stokes and the energy equations in terms of pressure $\bar{P}$, velocity $(\bar{u}, \bar{v}, \bar{w})$ and the temperature $\bar{T}$ of the fluid which are two-dimensional

$$
\begin{gathered}
\frac{\partial \bar{u}}{\partial \bar{x}}+\frac{\partial \bar{v}}{\partial \bar{y}}=0 \\
\bar{u} \frac{\partial \bar{u}}{\partial \bar{x}}+\bar{v} \frac{\partial \bar{u}}{\partial \bar{y}}=-\frac{1}{\rho} \frac{\partial \bar{P}}{\partial \bar{x}}+\nu\left(\frac{\partial^{2} \bar{u}}{\partial \bar{x}^{2}}+\frac{\partial^{2} \bar{u}}{\partial \bar{y}^{2}}\right)+\frac{\mu_{0} \bar{M}}{\rho} \frac{\partial \bar{H}}{\partial \bar{x}} \\
\bar{u} \frac{\partial \bar{v}}{\partial \bar{x}}+\bar{v} \frac{\partial \bar{v}}{\partial \bar{y}}=-\frac{1}{\rho} \frac{\partial \bar{P}}{\partial \bar{y}}+\nu\left(\frac{\partial^{2} \bar{v}}{\partial \bar{x}^{2}}+\frac{\partial^{2} \bar{v}}{\partial \bar{y}^{2}}\right)+\frac{\mu_{0} \bar{M}}{\rho} \frac{\partial \bar{H}}{\partial \bar{y}}+g \beta\left(\bar{T}-T_{\text {cold }}\right) \\
\bar{u} \frac{\partial \bar{w}}{\partial \bar{x}}+\bar{v} \frac{\partial \bar{w}}{\partial \bar{y}}=-\frac{1}{\rho} \frac{\partial \bar{P}}{\partial \bar{z}}+\nu\left(\frac{\partial^{2} \bar{w}}{\partial \bar{x}^{2}}+\frac{\partial^{2} \bar{w}}{\partial \bar{y}^{2}}\right) \\
\left(\bar{u} \frac{\partial \bar{T}}{\partial \bar{x}}+\bar{v} \frac{\partial \bar{T}}{\partial \bar{y}}\right)=-\mu_{0} \bar{T} \frac{\partial \bar{M}}{\partial \bar{T}}\left(\bar{u} \frac{\partial \bar{H}}{\partial \bar{x}}+\bar{v} \frac{\partial \bar{H}}{\partial \bar{y}}\right)+k\left(\frac{\partial^{2} \bar{T}}{\partial \bar{x}^{2}}+\frac{\partial^{2} \bar{T}}{\partial \bar{y}^{2}}\right) \\
\left.+2\left(\frac{\partial \bar{u}}{\partial \bar{x}}\right)^{2}+2\left(\frac{\partial \bar{v}}{\partial \bar{y}}\right)^{2}+\left(\frac{\partial \bar{v}}{\partial \bar{x}}+\frac{\partial \bar{u}}{\partial \bar{y}}\right)^{2}\right] .
\end{gathered}
$$

Here, $\mu, \nu, \rho, c_{p}, k$ and $\beta$ are the dynamic viscosity, the kinematic viscosity, the density, the specific heat, the thermal conductivity and the thermal expansion coefficient of the fluid, $\mu_{0}$ is the magnetic permeability of vacuum and $g$ is the magnitude of the gravitational acceleration.

$\bar{H}$ is the magnetic field strength and $\bar{M}=\chi \bar{H}\left(T_{c}-\bar{T}\right)$ is the magnetization where $\chi$ is the magnetic susceptibility of the blood and $T_{c}$ is the Curie temperature of iron.

The terms $\frac{\mu_{0} \bar{M}}{\rho} \frac{\partial \bar{H}}{\partial \bar{x}}$ and $\frac{\mu_{0} \bar{M}}{\rho} \frac{\partial \bar{H}}{\partial \bar{y}}$ are the components of the magnetization force. $g \beta\left(\bar{T}-T_{\text {cold }}\right)$ is the buoyancy force, where $T_{\text {cold }}$ is the temperature of the cold wall. The second term in the energy equation is the heating due to magnetization and the last term is the viscous dissipation which is a heat source caused by the friction between the fluid particles.

In fully developed flows the pressure is split as in [18]

$$
\begin{gathered}
\bar{P}(\bar{x}, \bar{y}, \bar{z})=\bar{p}(\bar{x}, \bar{y})+\bar{P}_{1}(\bar{z}) \\
\frac{\partial \bar{P}}{\partial \bar{z}}=\frac{\partial \bar{P}_{1}}{\partial \bar{z}}=\bar{P}_{z}=\text { constant } .
\end{gathered}
$$

The components of the magnetic field $\overrightarrow{\vec{H}}=\left(\bar{H}_{x}, \bar{H}_{y}\right)$ generated by an infinitely long electric wire is defined as in [19]

$$
\bar{H}_{x}=\frac{-I}{2 \pi} \frac{\bar{y}-\bar{b}}{(\bar{x}-\bar{a})^{2}+(\bar{y}-\bar{b})^{2}}, \quad \bar{H}_{y}=\frac{I}{2 \pi} \frac{\bar{x}-\bar{a}}{(\bar{x}-\bar{a})^{2}+(\bar{y}-\bar{b})^{2}}
$$


where $I$ is the electric current, $(\bar{a}, \bar{b})$ is the place of the point magnetic source. For square cavity $(\bar{a}, \bar{b})=(h / 2,-\bar{c})$ and for circular cavity $(\bar{a}, \bar{b})=(0,-h-$ $\bar{c})$. Here, $\bar{c}$ denotes the distance between the source and the cavity. Then, magnetic field strength is

$$
\bar{H}=\sqrt{\bar{H}_{x}^{2}+\bar{H}_{y}^{2}}=\frac{I}{2 \pi} \frac{1}{\sqrt{(\bar{x}-\bar{a})^{2}+(\bar{y}-\bar{b})^{2}}}
$$

For simplicity the non-dimensional variables are given as

$$
\begin{gathered}
x=\frac{\bar{x}}{h}, \quad y=\frac{\bar{y}}{h}, \quad z=\frac{\bar{z}}{h}, \quad u=\frac{\bar{u} h}{\nu}, \quad v=\frac{\bar{v} h}{\nu}, \quad w=\frac{\bar{w} h}{\nu}, \quad P=\frac{\bar{P} h^{2}}{\rho \nu^{2}}, \\
H=\frac{\bar{H}}{H_{0}}, \quad T=\frac{\bar{T}-T_{\text {cold }}}{T_{\text {hot }}-T_{\text {cold }}}
\end{gathered}
$$

where $h$ is the width of the square cavity and the radius of the circular cavity, $H_{0}$ is the magnetic field strength at $(h / 2,0)$ for square and $(0,-h)$ for circular cavity. $T_{\text {hot }}$ is the temperature of the hot wall. The reference values for the physical properties are $\rho=1050 \mathrm{kgm}^{-3}, \mu=3.2 \times 10^{-3} \mathrm{kgm}^{-1} \mathrm{~s}^{-1}, T_{\text {hot }}=$ $316.15^{\circ} \mathrm{K}, T_{\text {cold }}=276.65^{\circ} \mathrm{K}$ as in [9] and $h=1.1 \times 10^{-3} \mathrm{~m}$.

The magnetic field strength $H$ in non-dimensional form for square cavity

$$
H(x, y)=\frac{|b|}{\sqrt{(x-a)^{2}+(y-b)^{2}}}
$$

and for circular cavity

$$
H(x, y)=\frac{|c|}{\sqrt{(x-a)^{2}+(y-b)^{2}}}
$$

where $(a, b)=(\bar{a} / h, \bar{b} / h)$ and $c=\bar{c} / h$.

Thus, the non-dimensional equations are

$$
\begin{gathered}
\frac{\partial u}{\partial x}+\frac{\partial v}{\partial y}=0 \\
\frac{\partial^{2} u}{\partial x^{2}}+\frac{\partial^{2} u}{\partial y^{2}}=\frac{\partial p}{\partial x}+u \frac{\partial u}{\partial x}+v \frac{\partial u}{\partial y}-M n\left(T_{c}-T\right) H \frac{\partial H}{\partial x} \\
\frac{\partial^{2} v}{\partial x^{2}}+\frac{\partial^{2} v}{\partial y^{2}}=\frac{\partial p}{\partial y}+u \frac{\partial v}{\partial x}+v \frac{\partial v}{\partial y}-M n\left(T_{c}-T\right) H \frac{\partial H}{\partial y}-\frac{R a}{P r} T \\
\frac{\partial^{2} w}{\partial x^{2}}+\frac{\partial^{2} w}{\partial y^{2}}=P_{z}+u \frac{\partial w}{\partial x}+v \frac{\partial w}{\partial y}
\end{gathered}
$$




$$
\begin{aligned}
\frac{\partial^{2} T}{\partial x^{2}}+\frac{\partial^{2} T}{\partial y^{2}}= & \operatorname{Pr}\left(u \frac{\partial T}{\partial x}+v \frac{\partial T}{\partial y}\right)-\operatorname{MnEcPr}(\epsilon+T) H\left(u \frac{\partial H}{\partial x}+v \frac{\partial H}{\partial y}\right) \\
& -E c \operatorname{Pr}\left(2\left(\frac{\partial u}{\partial x}\right)^{2}+2\left(\frac{\partial v}{\partial y}\right)^{2}+\left(\frac{\partial v}{\partial x}+\frac{\partial u}{\partial y}\right)^{2}\right)
\end{aligned}
$$

In order to simulate the flow in terms of velocity and the pressure of the fluid pressure equation is derived by differentiating the $x-$ and

$y$-components of Navier-Stokes equations and adding them with the use of continuity equation

$$
\begin{aligned}
\frac{\partial^{2} p}{\partial x^{2}}+\frac{\partial^{2} p}{\partial y^{2}}= & \frac{R a}{\operatorname{Pr}} \frac{\partial T}{\partial y}-\left(\frac{\partial u}{\partial x}\right)^{2}-\left(\frac{\partial v}{\partial y}\right)^{2}-2 \frac{\partial v}{\partial x} \frac{\partial u}{\partial y} \\
& -M n\left(\frac{\partial T}{\partial x} H \frac{\partial H}{\partial x}+\frac{\partial T}{\partial y} H \frac{\partial H}{\partial y}\right) \\
& +M n\left(T_{c}-T\right)\left(\left(\frac{\partial H}{\partial x}\right)^{2}+\left(\frac{\partial H}{\partial y}\right)^{2}+H \nabla^{2} H\right) .
\end{aligned}
$$

The non-dimensional parameters entering the problem are

$$
\begin{gathered}
M n=\frac{\mu_{0} \chi H_{0}^{2}\left(T_{h o t}-T_{\text {cold }}\right) h^{2}}{\nu^{2} \rho} \text { (Magnetic number) } \\
\operatorname{Pr}=\frac{\rho c_{p} \nu}{k} \text { (Prandtl number) } \\
R a=\frac{g \rho c_{p} \beta\left(T_{h o t}-T_{\text {cold }}\right) h^{3}}{\nu k} \text { (Rayleigh number) } \\
\left.\epsilon=\frac{T_{\text {cold }}}{T_{h o t}-T_{\text {cold }}} \text { (Temperature number }\right) \\
\left.E c=\frac{\nu^{2}}{h^{2} c_{p}\left(T_{h o t}-T_{\text {cold }}\right)} \text { (Eckert number }\right) .
\end{gathered}
$$

Magnetic number expresses the ratio of the magnetic forces and the inertia forces. Prandtl number is the ratio of the momentum and thermal diffusivities. Rayleigh number is the product of the Grashof number and the Prandtl number where Grashof number denotes the ratio of the buoyancy forces to viscous forces. Eckert number defines the kinetic energy of the flow relative to the boundary layer enthalpy difference.

$2 \mathrm{D}$ stream function is introduced satisfying the continuity equation as $u=$ $\frac{\partial \Psi}{\partial y}, \quad v=-\frac{\partial \Psi}{\partial x}$. To visualize the flow pattern in the cavity, the stream function equation is obtained

$$
\frac{\partial^{2} \Psi}{\partial x^{2}}+\frac{\partial^{2} \Psi}{\partial y^{2}}=\frac{\partial u}{\partial y}-\frac{\partial v}{\partial x}
$$


The walls of the cavities are motionless and the velocity has no-slip boundary conditions. The square cavity is heated from the top and cooled from the bottom walls, horizontal walls are adiabatic. The circular cavity has no adiabatic walls. The pressure boundary conditions are approximated through $x-$ and $y$-components of the momentum equations using a forward difference for the pressure gradients and the DRBEM coordinate matrix $\mathbf{F}$ for all the other terms.

The problem geometry and the boundary conditions are presented in Figure 1 for both square and circular cavities.

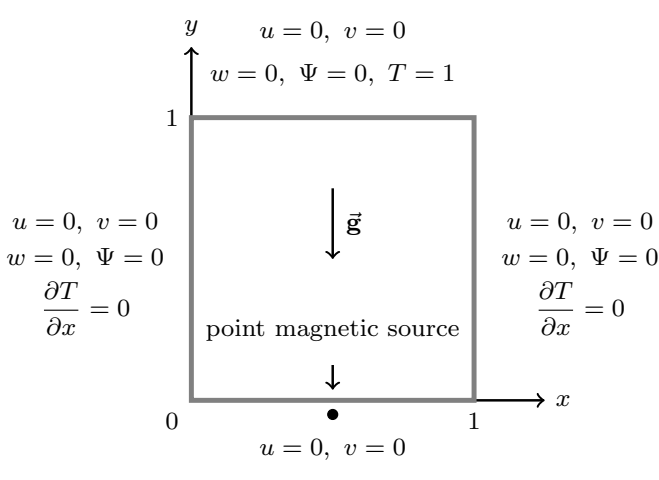

$w=0, \Psi=0, T=0$

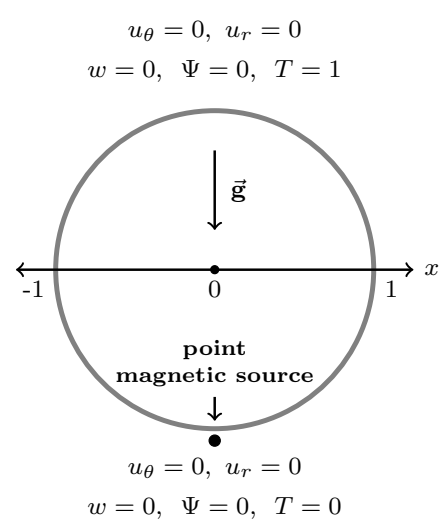

Figure 1: Problem geometry and boundary conditions

Eqs. (14)-(18) and (24) together with the mentioned boundary conditions define the two-dimensional flow and heat transfer of biomagnetic fluid (blood) in the cross-section of a long pipe, and the axial velocity.

\section{Application of DRBEM}

The dual reciprocity boundary element method is used to transform Eqs. (14)(18) and (24) into the boundary integral equations using the fundamental solution of Laplace equation $\left(u^{*}=(1 / 2 \pi) \ln (1 / r)\right)[13]$. The main advantage of the method is the reduction of problem dimension. It enables one to discretize only the boundary of the problem domain. Taking all the terms other than Laplacian as inhomogeneity, weighting the equations with the fundamental solution $u^{*}$ and using the Green's first identity two times an integral equation 
for the unknown is obtained

$$
c_{i} R_{i}+\int_{\Gamma} R q^{*} d \Gamma-\int_{\Gamma} u^{*} \frac{\partial R}{\partial n} d \Gamma=-\int_{\Omega} b_{R} u^{*} d \Omega
$$

where $R$ denotes $u, v, w, T, p$ or $\Psi$ and $b_{R}$ is the right-hand side of each corresponding equation for $R . \Gamma=\partial \Omega, q^{*}=\partial u^{*} / \partial n$ and $n$ is the outward unit normal to the boundary. $c_{i}=1 / 2$ for the boundary and $c_{i}=1$ for the interior nodes.

To eliminate the domain integral on the right-hand side of Eq. $(25) b_{R}$ is approximated by a linear radial basis function $f_{j}(r)=1+r_{j}$ which is connected to the particular solution $\hat{u}_{j}$ as $\nabla^{2} \hat{u}_{j}=f_{j},[13]$ and $r_{j}$ is the distance between the source and field points. Then,

$$
b_{R}=\sum_{j=1}^{N+L}\left(\alpha_{R}\right)_{j} \nabla^{2} \hat{u}_{j}
$$

where $\left(\alpha_{R}\right)_{j}$ 's are the undetermined coefficients for the approximation of the right-hand side $b_{R} . \quad N$ and $L$ are the number of boundary and the interior nodes, respectively.

Green's first identity is applied to the right-hand side of Eq. (25) and the boundary is discretized with $N$ constant elements to achieve a boundary integral equation for the unknown $R$.

$$
\begin{aligned}
c_{i} R_{i}+\sum_{k=1}^{N} \int_{\Gamma_{k}} R q^{*} d \Gamma-\sum_{k=1}^{N} \int_{\Gamma_{k}} u^{*} \frac{\partial R}{\partial n} d \Gamma= & \sum_{j=1}^{N+L}\left(\alpha_{R}\right)_{j}\left(c_{i} \hat{u}_{i j}+\sum_{k=1}^{N} \int_{\Gamma_{k}} \hat{u}_{j} q^{*} d \Gamma\right. \\
& \left.-\sum_{k=1}^{N} \int_{\Gamma_{k}} u^{*} \frac{\partial \hat{u}_{j}}{\partial n} d \Gamma\right) .
\end{aligned}
$$

Eq. (26) is used to determine the unknown coefficients $\left(\alpha_{R}\right)_{j}$ 's in terms of the DRBEM coordinate matrix $\mathbf{F}$ which is constructed by taking radial basis functions $f_{j}$ 's as columns

$$
\alpha_{R}=\mathbf{F}^{-1} b_{R}
$$

Writing Eq. (27) for all boundary and $L$ interior nodes and using Eq. (28) DRBEM discretized matrix-vector equations are obtained

$$
\begin{gathered}
\mathbf{H} u-\mathbf{G} \frac{\partial u}{\partial n}=(\mathbf{H} \hat{\mathbf{U}}-\mathbf{G} \hat{\mathbf{Q}}) \mathbf{F}^{-1}\left\{\frac{\partial p}{\partial x}+u \frac{\partial u}{\partial x}+v \frac{\partial u}{\partial y}-M n\left(T_{c}-T\right) H \frac{\partial H}{\partial x}\right\} \\
\mathbf{H} v-\mathbf{G} \frac{\partial v}{\partial n}=(\mathbf{H} \hat{\mathbf{U}}-\mathbf{G} \hat{\mathbf{Q}}) \mathbf{F}^{-1}\left\{\frac{\partial p}{\partial y}+u \frac{\partial v}{\partial x}+v \frac{\partial v}{\partial y}-M n\left(T_{c}-T\right) H \frac{\partial H}{\partial y}-\frac{R a}{P r} T\right\}
\end{gathered}
$$




$$
\begin{gathered}
\mathbf{H} w-\mathbf{G} \frac{\partial w}{\partial n}=(\mathbf{H} \hat{\mathbf{U}}-\mathbf{G} \hat{\mathbf{Q}}) \mathbf{F}^{-1}\left\{P_{z}+u \frac{\partial w}{\partial x}+v \frac{\partial w}{\partial y}\right\} \\
\mathbf{H} T-\mathbf{G} \frac{\partial T}{\partial n}=(\mathbf{H} \hat{\mathbf{U}}-\mathbf{G} \hat{\mathbf{Q}}) \mathbf{F}^{-1}\left\{\operatorname{Pr}\left(u \frac{\partial T}{\partial x}+v \frac{\partial T}{\partial y}\right)\right. \\
-M n E c \operatorname{Pr}(\epsilon+T) H\left(u \frac{\partial H}{\partial x}+v \frac{\partial H}{\partial y}\right) \\
\left.-\operatorname{Ec} \operatorname{Pr}\left(2\left(\frac{\partial u}{\partial x}\right)^{2}+2\left(\frac{\partial v}{\partial y}\right)^{2}+\left(\frac{\partial v}{\partial x}+\frac{\partial u}{\partial y}\right)^{2}\right)\right\} \\
\mathbf{H} p-\mathbf{G} \frac{\partial p}{\partial n}=(\mathbf{H} \hat{\mathbf{U}}-\mathbf{G} \hat{\mathbf{Q}}) \mathbf{F}^{-1}\left\{\frac{R a}{P r} \frac{\partial T}{\partial y}-\left(\frac{\partial u}{\partial x}\right)^{2}-\left(\frac{\partial v}{\partial y}\right)^{2}-2 \frac{\partial v}{\partial x} \frac{\partial u}{\partial y}\right. \\
-M n\left(\frac{\partial T}{\partial x} H \frac{\partial H}{\partial x}+\frac{\partial T}{\partial y} H \frac{\partial H}{\partial y}\right) \\
\left.+M n\left(T_{c}-T\right)\left(\left(\frac{\partial H}{\partial x}\right)^{2}+\left(\frac{\partial H}{\partial y}\right)^{2}+H \nabla^{2} H\right)\right\} \\
\mathbf{H} \Psi-\mathbf{G} \frac{\partial \Psi}{\partial n}=(\mathbf{H} \hat{\mathbf{U}}-\mathbf{G} \hat{\mathbf{Q}}) \mathbf{F}^{-1}\left\{\frac{\partial u}{\partial y}-\frac{\partial v}{\partial x}\right\}
\end{gathered}
$$

where

$$
\begin{gathered}
\mathbf{H}_{i j}=c_{i} \delta_{i j}+\int_{\Gamma_{j}} q^{*} d \Gamma_{j}, \quad \mathbf{H}_{i i}=c_{i}, \\
\mathbf{G}_{i j}=\int_{\Gamma_{j}} u^{*} d \Gamma_{j}, \quad \mathbf{G}_{i i}=\frac{l}{2 \pi}\left(\ln \left(\frac{2}{l}\right)+1\right)
\end{gathered}
$$

$l$ is the length of the element.

The matrices $\hat{\mathbf{U}}, \hat{\mathbf{Q}}$ are constructed by taking each vector $\hat{u}_{j}$ and $\hat{q}_{j}=\partial \hat{u}_{j} / \partial n$ as columns, respectively.

The spatial derivatives of the unknowns on the right-hand sides of Eqs. (29)(34) are approximated by using DRBEM coordinate matrix $\mathbf{F}$ as

$$
\frac{\partial R}{\partial \eta}=\frac{\partial \mathbf{F}}{\partial \eta} \mathbf{F}^{-1} R, \quad \frac{\partial^{2} R}{\partial \xi \partial \eta}=\frac{\partial \mathbf{F}}{\partial \xi} \mathbf{F}^{-1} \frac{\partial \mathbf{F}}{\partial \eta} \mathbf{F}^{-1} R
$$

with $\eta$, $\xi$ being $x$ or $y$.

The discretized system of Eqs. (29)-(34) are solved iteratively by taking initially

$$
u^{(0)}=v^{(0)}=0 \text { in } \Omega \cup \Gamma
$$

and

$$
T^{(0)}= \begin{cases}1 & \text { on the hot boundary } \\ 0 & \text { otherwise }\end{cases}
$$


In the stagnant situation of the fluid the transverse pressure gradient forces are balanced by the effect of the magnetic and buoyancy forces hence, initial pressure gradients are

$$
\begin{aligned}
& \frac{\partial p}{\partial x}^{(0)}=\operatorname{Mn}\left(T-T_{c}\right) H\left(\frac{\partial H}{\partial x}\right)+10^{-12} \\
& \frac{\partial p}{\partial y}^{(0)}=\operatorname{Mn}\left(T-T_{c}\right) H\left(\frac{\partial H}{\partial y}\right)+\frac{R a}{P r} T+10^{-12} .
\end{aligned}
$$

The system of Eqs. (29)-(34) with the boundary conditions specified on Figure 1 are solved iteratively starting from Eq. (29) and relaxing temperature and pressure values with relaxation parameters in $(0,1)$ to accelerate the convergence. The iteration continues until a preassigned tolerance is reached. The stopping criteria for the iteration is

$$
\frac{\left\|R^{(n+1)}-R^{(n)}\right\|_{\infty}}{\left\|R^{(n)}\right\|_{\infty}}<10^{-3}
$$

where $R$ denotes $u, v, w, p, T$ or $\Psi$ and $n$ is the iteration number.

\section{Numerical Results and Discussions}

Convection flow of blood in square and circular cavities under point magnetic source are investigated by using DRBEM. The physical properties of blood presented in [9] are considered to set the dimensionless parameters. $\operatorname{Pr}=20$, $E c=1.25 \times 10^{-8}, \epsilon=7$ are taken. The pressure gradient given to the fluid in the axial direction is $P_{z}=-8000$ [2] and $c=0.05$. The proposed numerical scheme and results are validated with the natural convection flow of air in a square cavity without magnetization force by taking $\operatorname{Pr}=0.7$ and $R a=10^{3}$. The obtained results are in good agreement with the ones in [20]. The flow is studied in a square cavity with adiabatic vertical walls. Both in square and circular cavities the top wall is hot and the bottom wall is cold.

\subsection{Convection of blood in a square cavity}

The effect of buoyancy force only on the flow $(M n=0)$ is seen in Figures 2and 3. The buoyancy force divides the flow into two vortices with centers on $y=0.5$ line. Pressure is highly concentrated at the top of the cavity showing a drop at the center. Horizontal velocity consists of four loops emanating from the corners and vertical velocity spreads through the cavity. The buoyancy force shifts the isotherms through the hot wall. Thus, an increase in Rayleigh number accelerates the planar velocities, increases the pressure in magnitude and squeezes the isotherms through the hot wall. 

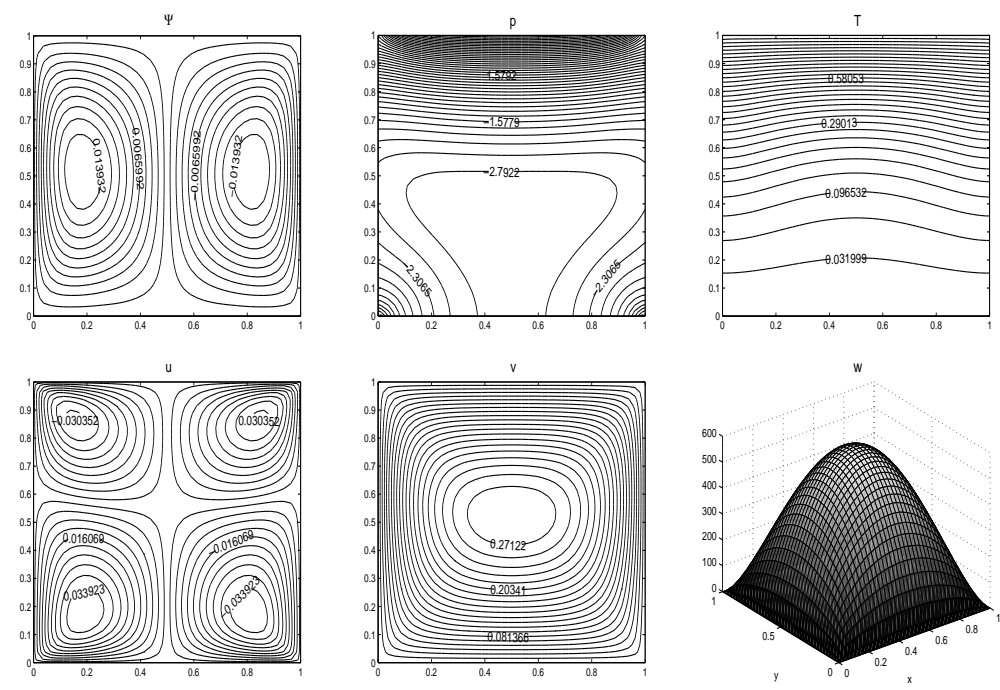

Figure 2: Viscous dissipation is neglected. $N=160, R a=10^{3}, M n=0$
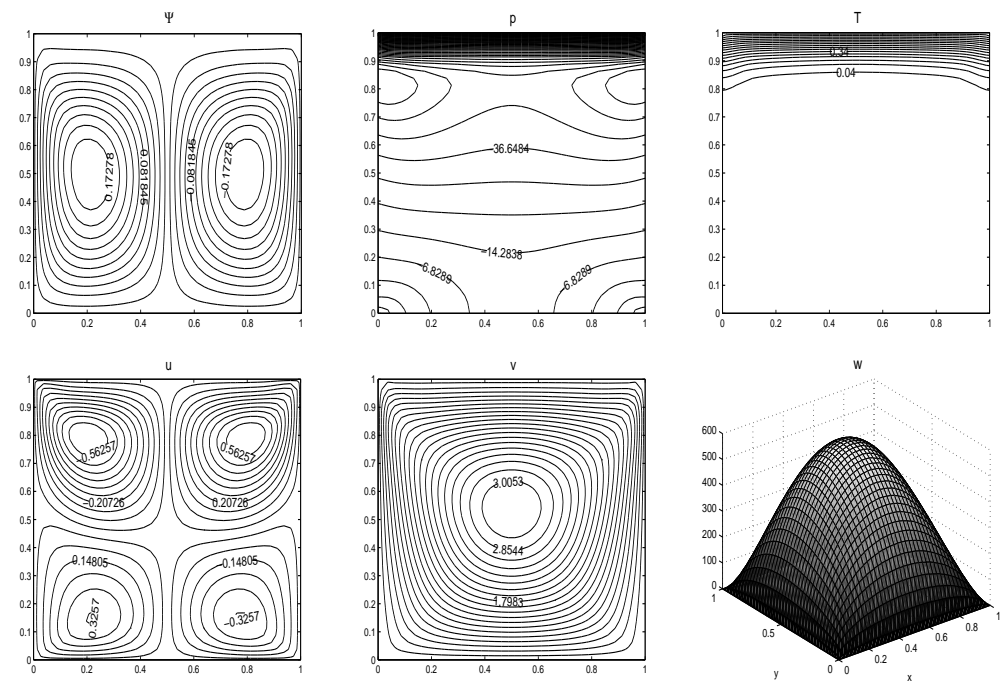

Figure 3: Viscous dissipation is neglected. $N=160, R a=10^{5}, M n=0$

Figures 4 and 5 display the effect of the magnetic source only on the flow 
profiles and the temperature of the fluid for $M n=5,200$ with $R a=0$. An increase in Magnetic number causes an increase in the magnitude of the planar velocities and the pressure. The axial velocity shows a flattening tendency around the point magnetic source and the pressure around the magnetic source extends through the cavity. Flow on the transverse plane is divided into two vortices rotating in opposite directions. A further increase in $M n$ moves the center of vortices through the magnetic source. The main effect of the point magnetic source below the cold wall is the cooling of the channel starting from the bottom wall. For values $M n \geq 80$ the fluid in the channel is completely cooled except a thin layer near the upper wall and secondary flows show up through bottom and top corners. $v$ - velocity shows the pushing effect of the magnetic point source and a thin boundary layer occurs just above and around the source.

The main effect of the magnetic source (and its increasing intensity) is to accelerate the fluid flow and increase the pressure on the fluid, and thus to cool down the channel through the upper wall. This is also the idea of cooling the head capsules of nuclear reactors.
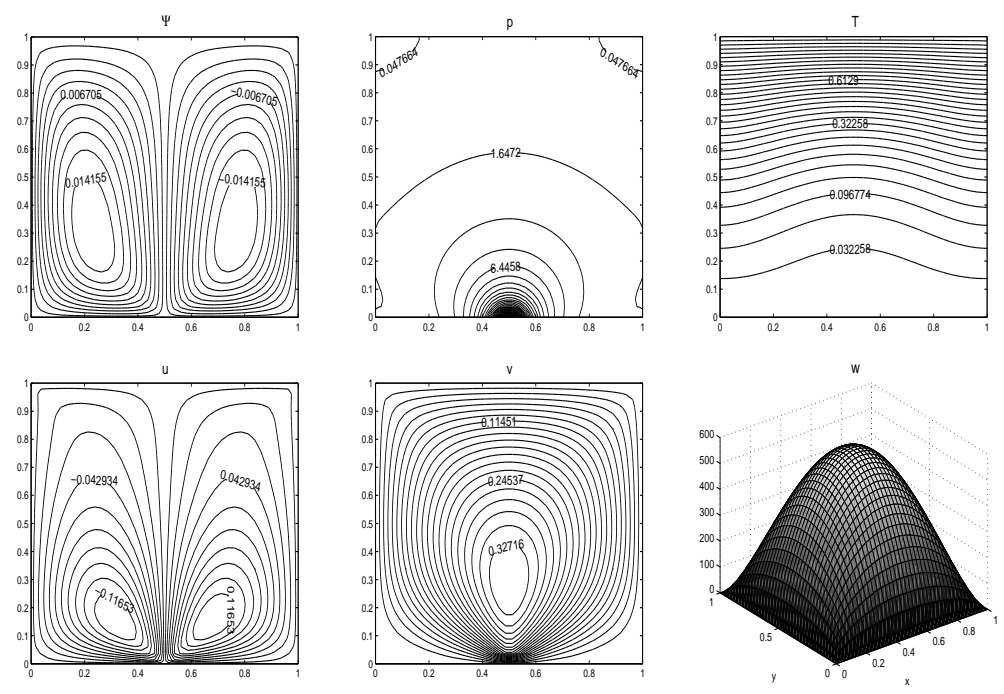

Figure 4: Viscous dissipation is neglected. $N=160, R a=0, M n=5$ 

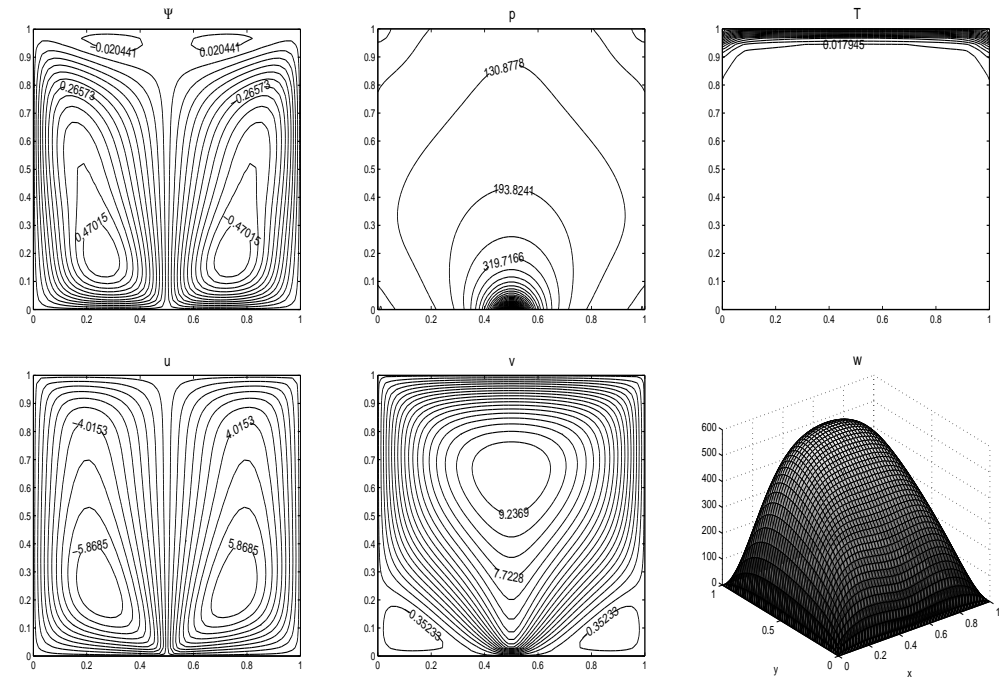

Figure 5: Viscous dissipation is neglected. $N=200, R a=0, M n=200$
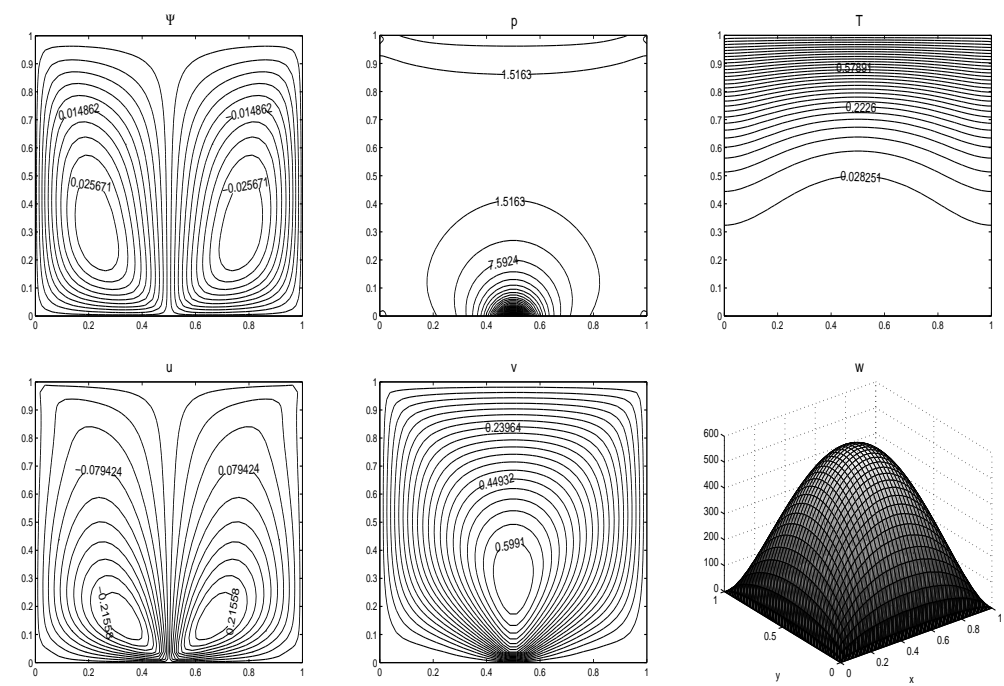

Figure 6: Viscous dissipation is neglected. $N=160, M n=10, R a=10^{3}$ 
Both the buoyancy force and magnetic source effects are seen $(M n=10$ and $R a=10^{3}, 10^{5}$ ) on Figures 6, 7. For small $M n$ the buoyancy effect starts to dominate the flow as $R a$ increases. When $R a \geq 10^{4}$ the thermal convection in the flow reduces the pushing effect of the magnetic source and viscous effect is reduced. As $R a$ increases the behavior of axial velocity stays the same since the $u$ - and $v$-velocities are not altered significantly.
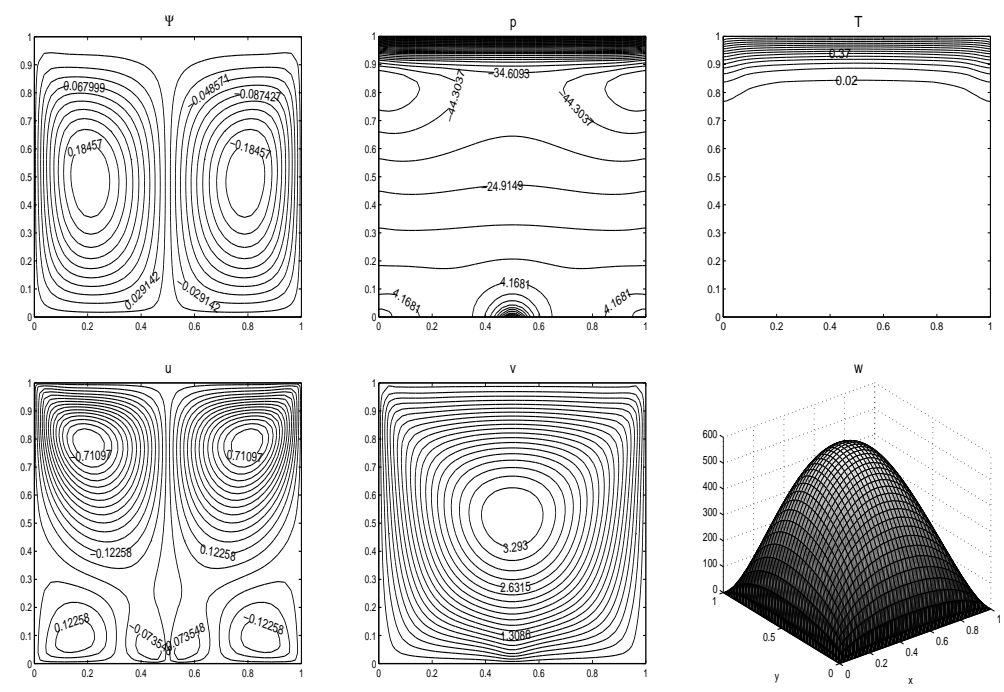

Figure 7: Viscous dissipation is neglected. $N=160, M n=10, R a=10^{5}$

When $M n=80$ is reached (Figures 8-9) the magnetization force dominates the buoyancy force up to $R a=10^{4}$. The influence of buoyancy force is observed in streamlines and pressure as the center of vortices move upwards and pressure starts to concentrate close to the top heated wall. But with an increase in magnetic number the cooling of the channel is much faster compared to an increase in Rayleigh number. 

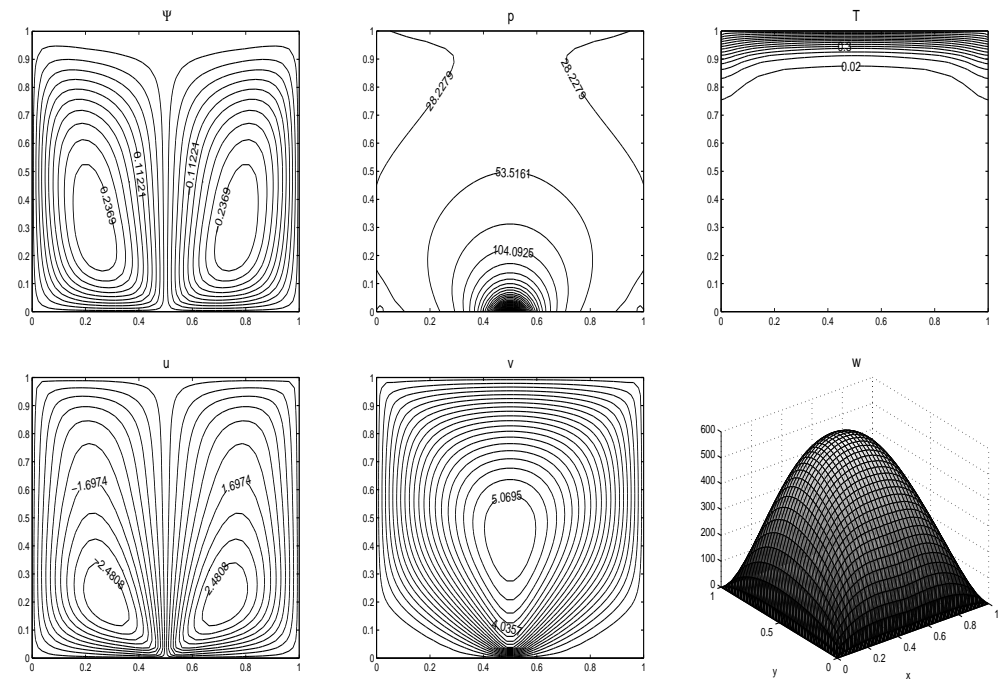

Figure 8: Viscous dissipation is neglected. $N=160, M n=80, R a=10^{4}$
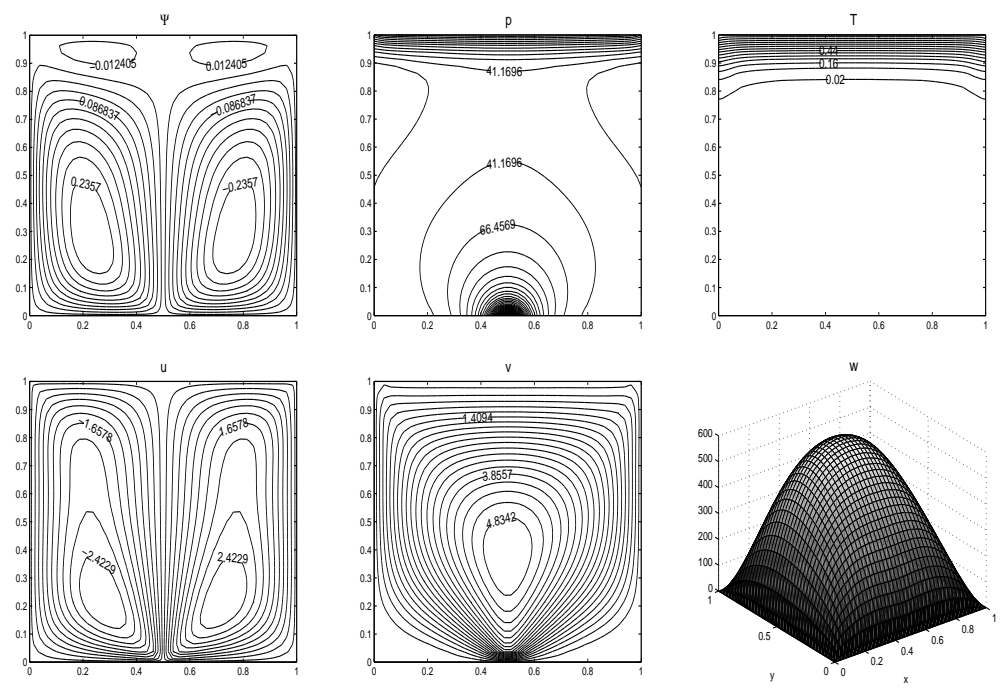

Figure 9: Viscous dissipation is neglected. $N=160, M n=80, R a=10^{5}$ 

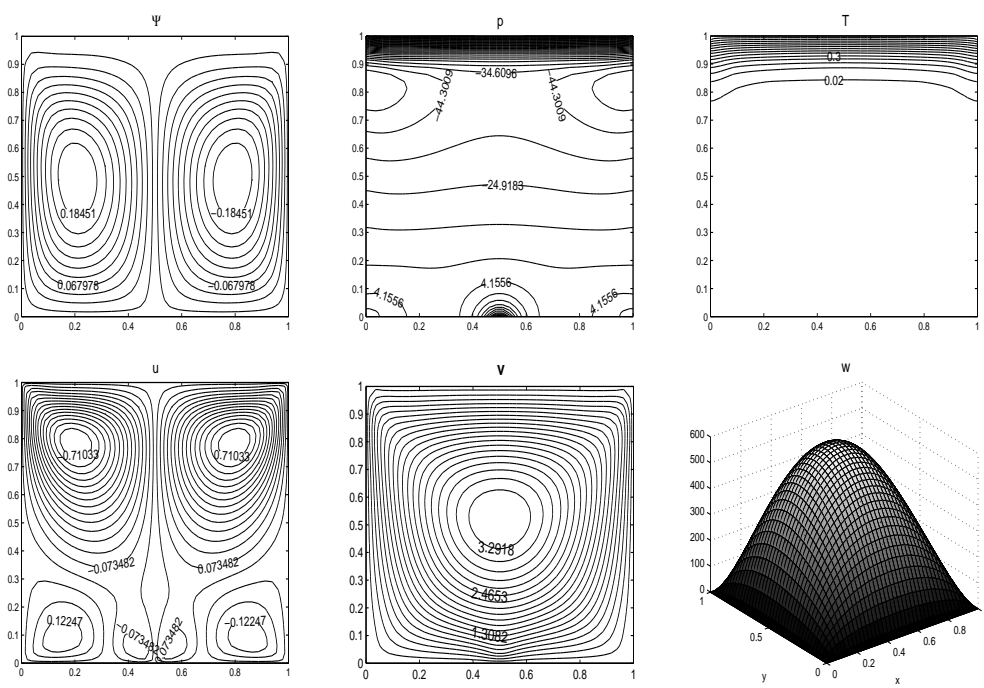

Figure 10: Viscous dissipation is added. $N=160, M n=10, R a=10^{5}$
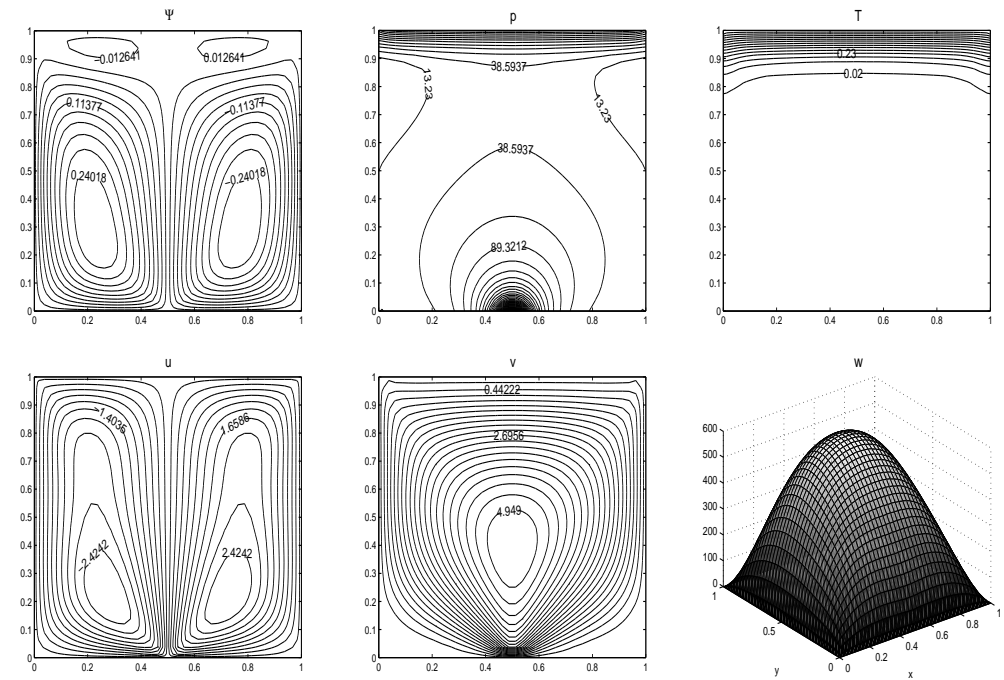

Figure 11: Viscous dissipation is added. $N=160, M n=80, R a=10^{5}$

Figures 10 and 11 show the behavior and heat exchange of a biomagnetic 
dissipative viscous fluid. It is observed that the viscous dissipation does not influence the flow and the heat transfer characteristics for small Rayleigh numbers. A slight difference is observed only when $R a=10^{5}$ comparing Figures 7,10 and 9,11 .

\subsection{Convection of blood in a circular cavity}

In this problem, the blood flow and its temperature disturbance is studied in a circular cavity heated on the upper half wall which is a more realistic case. The influence of buoyancy force is the acceleration of planar velocities as in the case of square cavity when $M n=0$. The pressure is concentrated around the hot wall and the discontinuity points since there is no adiabatic wall. The cavity is nearly cooled down for $R a \approx 10^{4}$ (Figure 12 ).
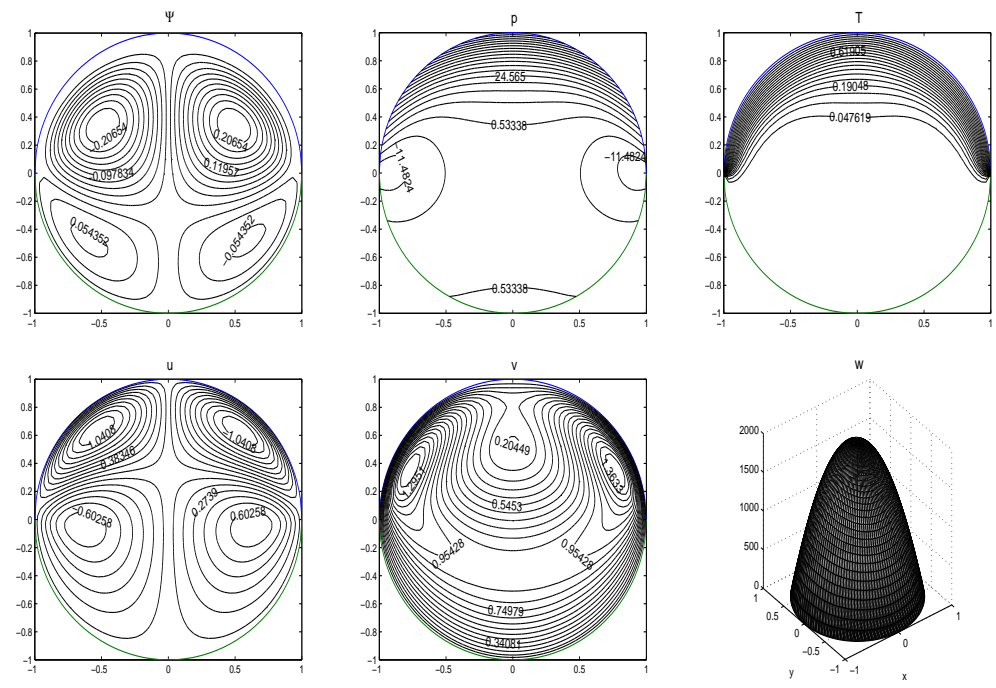

Figure 12: Viscous dissipation is neglected. $N=120, M n=0, R a=10^{4}$

Figures 13-14 display the velocity, pressure and the temperature variations for increasing magnetic effect $M n=10$ and 30 when $R a=0$. The flow behavior is similar to the square cavity case. Increasing magnetic field intensity accelerates the flow in the cavity and decelerates it in the axial direction. The heat transfers directly between the hot and cold walls. Pressure increases and center of vortices move through the source point. 

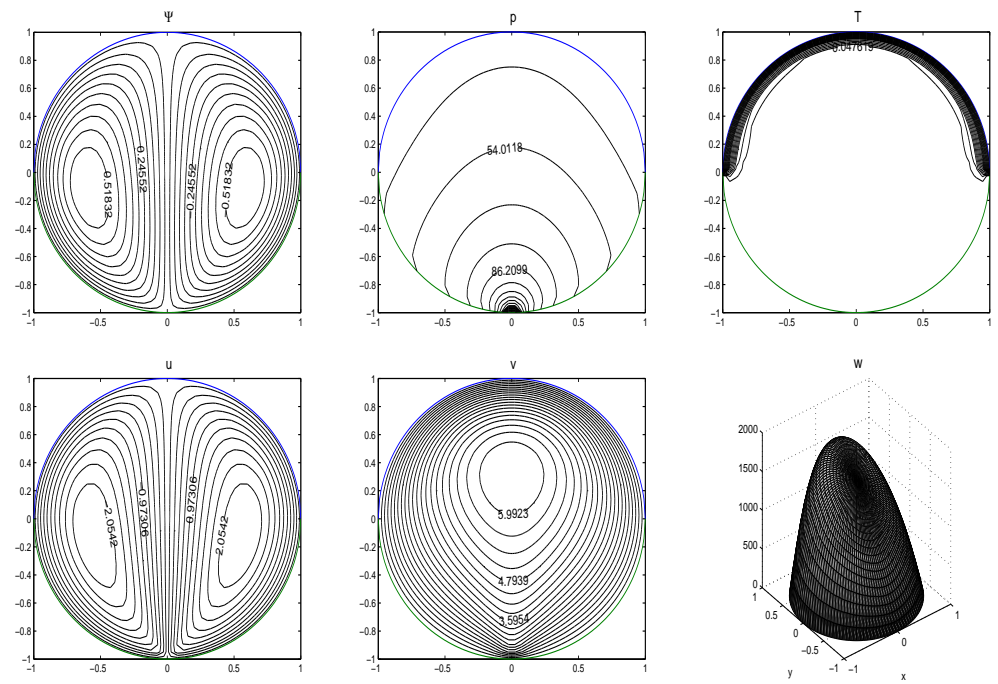

Figure 13: Viscous dissipation is neglected. $N=120, M n=10, R a=0$
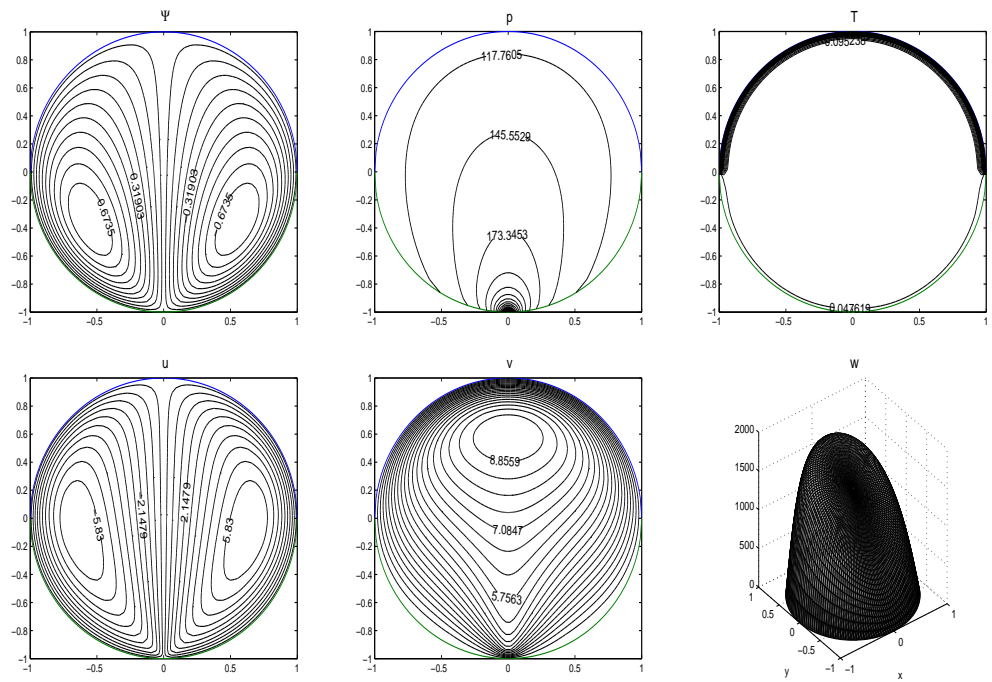

Figure 14: Viscous dissipation is neglected. $N=160, M n=30, R a=0$ 

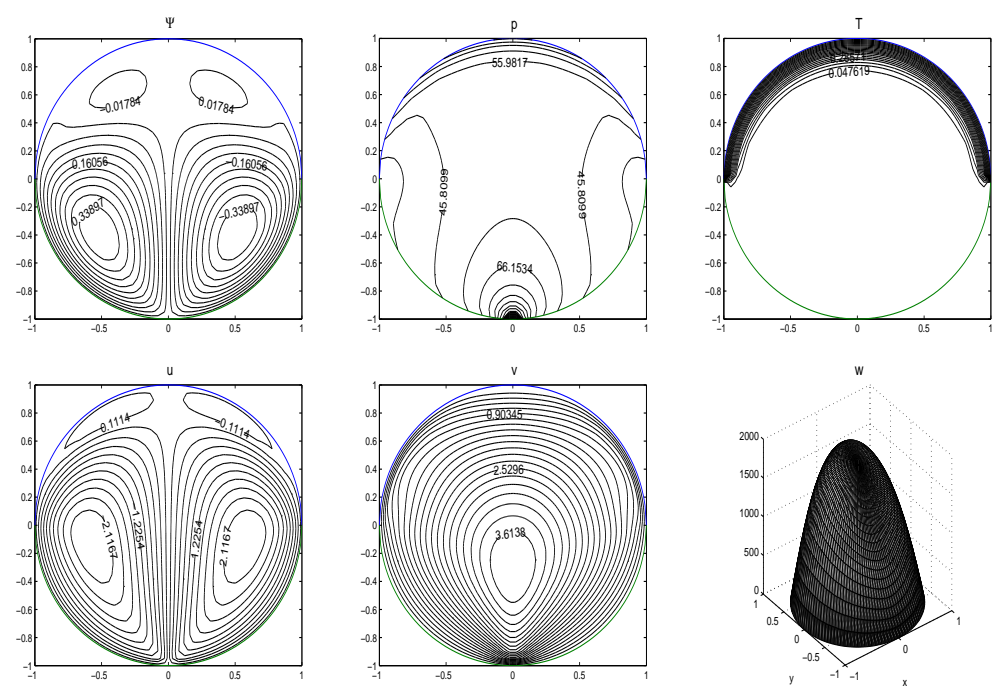

Figure 15: Viscous dissipation is neglected. $N=120, M n=10, R a=10^{4}$
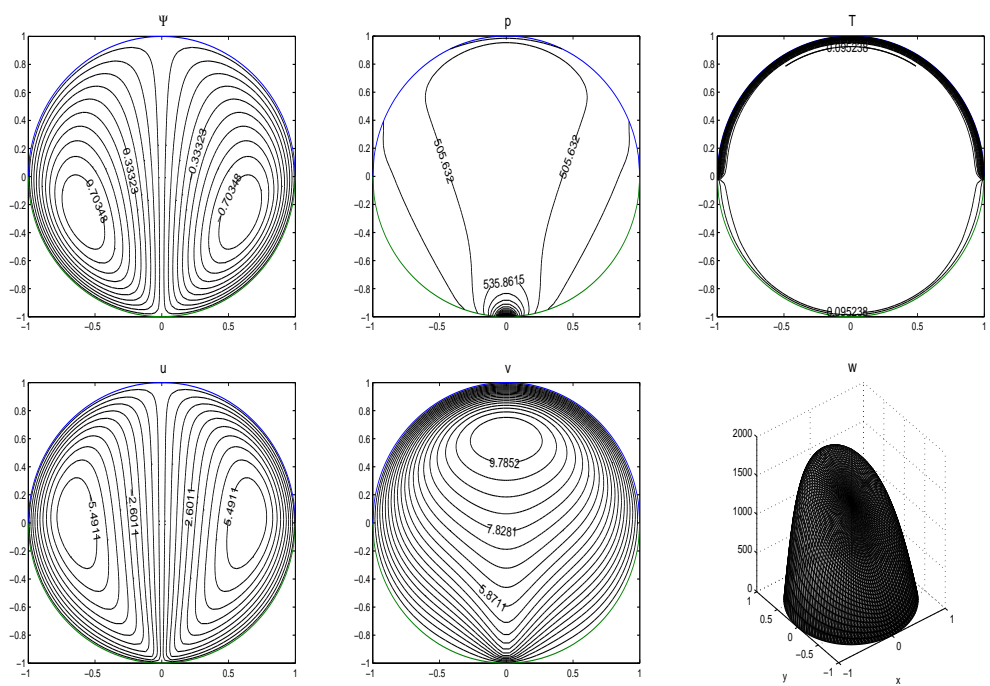

Figure 16: Viscous dissipation is neglected. $N=120, M n=50, R a=10^{4}$

When both magnetic source and buoyancy force are present the buoyancy 
force dominates the magnetization force when $R a=10^{4}$ is reached for small $M n=10$ (Figure 15). But, for larger $M n$ values the blood flow and its heat exchange is under the control of magnetic source up to $R a=10^{4}$. (Figure 16) The viscous dissipation effect on the flow in a circular cavity is not observed due to the sudden passage from the hot wall to the cold wall for the ranges of magnetic number and Rayleigh number values considered.

\section{Conclusion}

The influence of the magnetization and the buoyancy forces on the blood flow in a long pipe with square and circular cross-sections is investigated. When the magnetic source is below the cold wall the main effect of the magnetization and buoyancy forces is to cool down the cavity. However, increasing magnetic field intensity is more effective than increasing buoyancy force in cooling the fluid. Increasing magnetic field intensity accelerates the flow in the cavity and decelerates it in the axial direction around the source. Viscous dissipation can be seen slightly only when $R a$ is high and it is lost for larger values of $M n$.

\section{References}

[1] Higashi T, Yamagishi A, Takeuchi T, Kawaguchi N, Sagawa S, Onishi $\mathrm{S}$, and Date M. Orientation of erythrocytes in a strong magnetic field. Blood, 82 (4):1328-1334, 1993.

[2] Tzirtzilakis EE, Sakalis VD, Kafoussias NG, and Hatzikonstantinou PM. Biomagnetic fluid flow in a $3 \mathrm{D}$ rectangular duct. International Journal for Numerical Methods in Fluids, 44:1279-1298, 2004.

[3] Tzirtzilakis EE. A mathematical model for blood flow in a magnetic field. Physics of Fluids, 17:077103:1-15, 2005.

[4] Kenjeres S. Numerical analysis of blood flow in realistic arteries subjected to strong non-uniform magnetic fields. International Journal for Heat and Fluid Flow, 29:752-764, 2008.

[5] Khashan SA, Elnajjar E, and Haik Y. Numerical simulation of the continuous biomagnetic separation in a two-dimensional channel. International Journal for Multiphase Flow, 37:947-955, 2011.

[6] Tzirakis K, Papaharilaou Y, Giordano D, and Ekaterinaris J. Numerical investigation of biomagnetic fluids in circular ducts. International Journal for Numerical Methods in Biomedical Engineering, 30:297-317, 2014. 
[7] Kabeel AE, El-Said EMS, and Dafea SA. A review of magnetic field effects on flow and heat transfer in liquids: Present status and future potential for studies and applications. Reneweble and Sustainable Reviews, 45:830837, 2015.

[8] Tzirtzilakis EE and Kafoussias NG. Biomagnetic fluid flow over a stretching sheet with non linear temperature dependent magnetization. $Z A M P$, 54:551-565, 2003.

[9] Loukopoulos VC and Tzirtzilakis EE. Biomagnetic channel flow in spatially varying magnetic field. International Journal of Engineering Science, 42:571-590, 2004.

[10] Morega A and Faur S. A FEM analysis of biomagnetic fluid flow in a rectangular duct under the influence of a magnetic field. In Proceedings of the Third Workshop on Mathematical Modelling of Environmental and Life Sciences Problems, pages 205-218, 2004.

[11] Alimohamadi H, Dehghan-Niri V, and Ashjaee M. Improvement of heat transfer performances of biomagnetic flow in a rectangular duct under different types of magnetic fields. International Journal of Technology Enhancements and Emerging Engineering Research, 2:44-48, 2014.

[12] Idris NA, Amin N, and Rahmat H. Effect of gravitational acceleration on unsteady biomagnetic fluid flow. Applied and Computational Mathematics, 3(6):285-294, 2014.

[13] Partridge PW, Brebbia CA, and Wrobel LC. The Dual Reciprocity Boundary Element Method. Computational Mechanics Publications, Sauthampton, Boston, 1992.

[14] Senel P and Tezer-Sezgin M. DRBEM solutions of Stokes and NavierStokes equations in cavities under point source magnetic field. Engineering Analysis with Boundary Elements, 64:158-175, 2016.

[15] Tezer-Sezgin M, Bozkaya C, and Turk O. BEM and FEM based numerical simulations for biomagnetic fluid flow. Engineering Analysis with Boundary Elements, 37:1127-1135, 2013.

[16] Senel P and Tezer-Sezgin M. DRBEM solution of biomagnetic fluid flow under a point source magnetic field. In Proceedings of the 16th International Conference on Computational and Mathematical Methods in Science and Engineering (CMMSE 2016), pages 1172-1183, 2016. 
[17] Lienhard IV JH and Lienhard V JH. A Heat Transfer Textbook. Cambridge, Massachussetts, 2000.

[18] Fletcher CAJ. Computational Techniques for Fluid Dynamics 2. Springer, Berlin, 1991.

[19] Plansey R and Collin RE. Principles and Applications of Electromagnetic Fields. Mc Graw-Hill, NewYork, 1961.

[20] Lo DC, Young DL, and Tsai CC. High resolution of 2D natural convection in a cavity by the DQ method. Journal of Computational and Applied Mathematics, 203(1):219-236, 2007.

P. Senel,

Department of Mathematics,

Middle East Technical University,

06800, Ankara, TURKEY.

Email: psenel44@gmail.com

M. Tezer-Sezgin,

Department of Mathematics,

Middle East Technical University,

06800, Ankara, TURKEY.

Email:munt@metu.edu.tr 